\title{
Water Fire Fighting System Semi-physical Simulation Based on Simulink's RTW
}

\author{
Yi Xianglie \\ College of Naval Architecture and Power \\ Naval Univ. of Engineering \\ Wuhan, China
}

\author{
Qiu Jinshui \\ College of Naval Architecture and Power \\ Naval Univ. of Engineering \\ Wuhan, China
}

\begin{abstract}
This paper presents an approach to integrate simulation model with Visual $\mathrm{C}++$ easily by translating simulation model to embedded $\mathrm{C}++$ code. Then the advantages of Simulink and Visual $\mathrm{C}++$ are fully used by this approach. According to a real example the development steps and realization of water fire fighting system based on this approach is discussed in detail.
\end{abstract}

Keywords- Seawater Fire-Fighting System, Simulink, RTW, $V C++$

\section{INTRODUCTION}

Currently, the market demand for the product shows diversity and trend of fast, which makes product development needs facing the contradiction between the rapid development and diversity, the concurrent engineering (design, implementation, testing and production preparation at the same time) was put on the the agenda. system design software (Simulink software) is used in Matlab language to create a new graphical modeling tool that eliminates the inefficiencies caused by the program code and complicated programming, you can easily create a system model (for example, the object-oriented system model, controller model), the simulation can be observed through the control algorithm performance; and, if the control algorithm is not ideal, you can quickly revise the design and repeated the experiment, until you find a better control scheme. As to specific implementations, the design of the control algorithm does not need to program directly, for the controller Simulink model is built up through the RTW tools which can turn the Simulink model into the $\mathrm{C} / \mathrm{C}++$ code which can run independently from the Matlab environment, download to memory, thereby greatly reducing the workload of the encoding software engineers. Simulink is a well-known graphical modeling and simulation tools; it can be electronic, circuit, control engineering, signal processing and other areas of simulation, with a convenient and flexible modeling features, much more widely used in control system design and simulation. The semi-physical simulation system needs to connect to a particular hardware, custom control software, as to the realization of the features it appears that it lack of capacity, in which the Visual $\mathrm{C}++$ (VC) is precisely adept. $\mathrm{VC}$ is a visual $\mathrm{C}++$ programming environment with a powerful and flexible hardware control features also has man-machine interface design features.

\section{ENVIRONMENT}

Water Fire Fighting System and Power System have a principle of similarity. From the 60s of last century Power system simulation technology has already fully developed. In fact, the fire water system simulation can be realized by the simulation of power system through using power system simulation software. the simulation environment used in the simulation are listed blow:

The simulation software has Matlab7.04, RTW6.2, Simulink6.2, Visual $\mathrm{C}++6.0$.Operating system is Windows $\mathrm{xp}$, computer-related configuration is as follows:

CPU: Intel ${ }^{\circledR}$ CoreTM 2 Duo Processer T7100(1.8GHz, $800 \mathrm{MHz}$ FSB, $2 \mathrm{MB}$ L2 cache)

HDD: 120GB Memory: 1GB DDR

\section{ASSUMPTIONS}

1) Assuming water is the ideal fluid, you can use Bernoulli equation in the analysis of its kinetics.

2) The water pressure generated by the height difference can be neglected.

3) All the main are of the same section area, and branch pipe's section is the same, fire hydrants are also of the same size.

4) Ignore the bend loss. According to Bernoulli's equation [1]:

$$
P+\rho V^{2} / 2+\rho \cdot g \cdot h=C
$$

We can get two conclusions:

1) Any measured point as long as connected with the fire pump; water pressure of the given point is equal to pressure of the fire pump;

2) The flux through any chosen section, as long as which is connected to the pump, is direct proportion to the area of the section. For instance, a water fire-fighting system as shown in Figure 1:

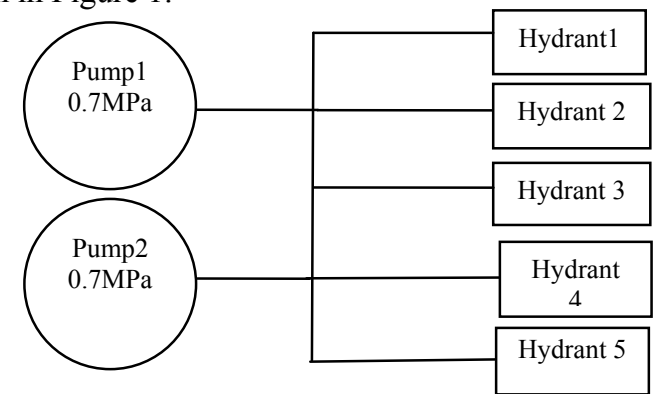

Figure 1. Firefighting system chart. 
Assume the pressure on the exit of pump1 is $0.7 \mathrm{MPa}$; flow flux it can provide is $350 \mathrm{~m}^{3} / \mathrm{h}$; and the pressure on the exit of pump2 is $0.7 \mathrm{MPa}$; flow flux it can provide is also $350 \mathrm{~m}^{3} / \mathrm{h}$; so according to the two inferences, we can get this: the pressure on each hydrant is $0.7 \mathrm{MPa}$, the flow flux of teach hydrant is $140 \mathrm{~m}^{3} / \mathrm{h}$.

In power systems, according to Ohm's law and the current law, voltage law:

$$
\begin{gathered}
I_{1}+I_{2}+I_{3}+\cdots \cdots=I \text { and } \\
U_{1}=U_{2}=U_{3}=\cdots \cdots=U
\end{gathered}
$$

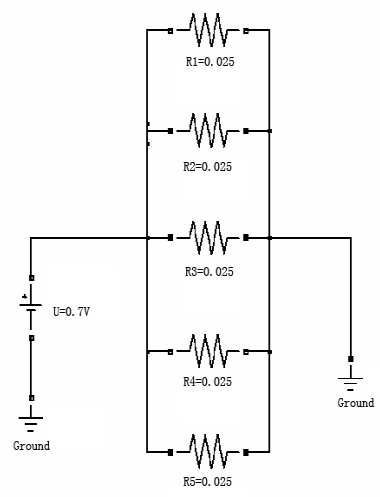

Figure 2. Power system graph.

According to the available laws related to the power system, the voltage of each resistor is $0.7 \mathrm{~V}$; the current through each resistor is $140 \mathrm{~A}$. If you want to do real-time simulation of water fire fighting system through the simulation of power systems, you have to use two sets of power system simulation system, for the circuit current is related to the resistance of the circuit. If you want to observe the water pressure on fire hydrants, Fire pump will be considered as a system voltage source; another set of power system the fire pump will be considered as a current source, to observe the flux through each hydrant.

\section{MODELING:}

1) Assume a fire pump as a voltage source or current source, the start button as a switch, as is shown in Figure 3:
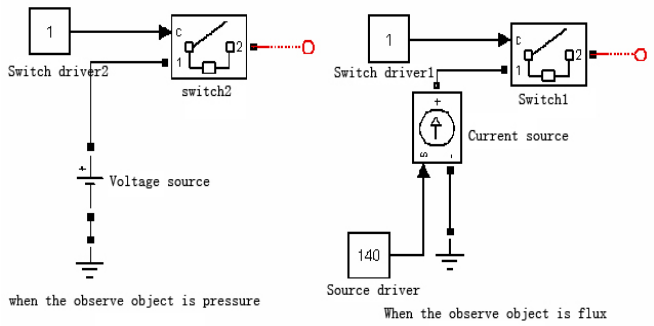

Figure 3. A power circuit equivalent to the firefighting pump.

2) Fire hydrant can be substituted with a resistor, which was shown in Figure 4.

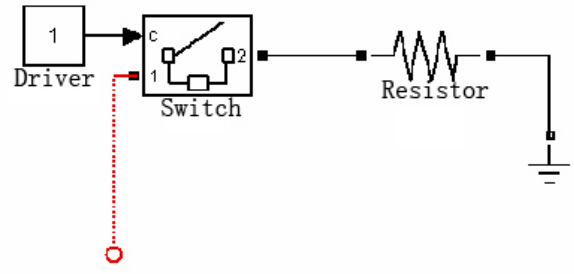

Figure 4. A power circuit equivalent to a fire hydrant

3) Pipes can be substituted with a wire instead;

4) Valve can be replaced by a switch,shown in Figure.5;

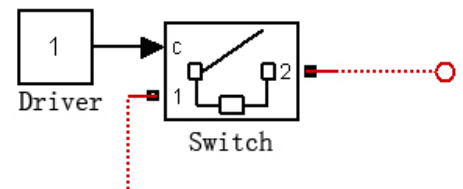

Figure 5. A power circuit equivalent to a valve

5) Pressure gauge replaced with a voltmeter,shown in Figure.6;

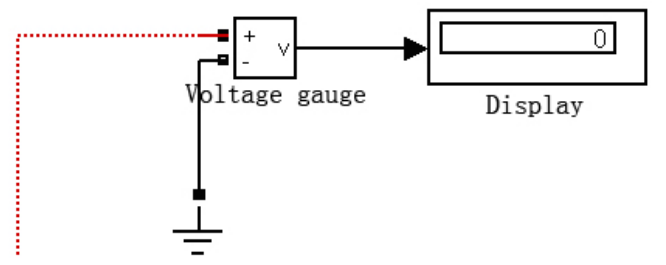

Figure 6. A power circuit equivalent to a pressure gauge

6) Flux meter replaced with a ammeter, shown in Figure.7;

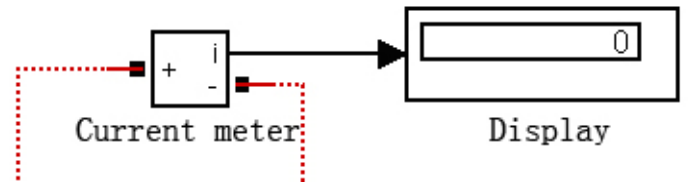

Figure 7. A power circuit equivalent to a flux meter

7) Damage hypothesis: pipe damage can be assumed as the corresponding wire switch to one end of the ground.

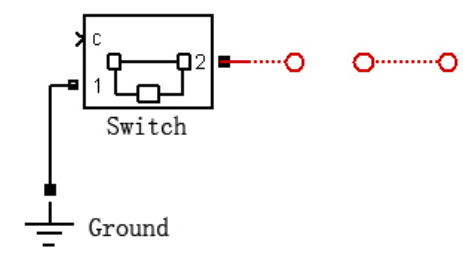

Figure 8. A power circuit equivalent to a pipe in damage status

8) Data show. if it is to consider flux, the fire pump will be replaced by the current source, current meter replace the flow meter. Consider the pressure, a voltage source will replace the fire pump, voltage meter replace the pressure gauge [2][3]. 


\section{Generating C + + CODE By the SimulinK's RTW}

According to the 8 definition in part 4 , you can create two power system structure diagram, after the program debugging passed, RTW will be able to put some parameters in the Simulink model after the targeted signal is set to global variables, the model automatically generated executable code of the running target system, you can easily interact with the Simulink model, for online parameter adjustment and signal communication. Using RTW system feature can be adjusted by the model, monitoring of parameters or signal which was set to global variables. Try to do the following:

Select in the Simulink model, set the signal, right click and select Signal Properties ... menu, the pop-up dialog box in Figure.9,

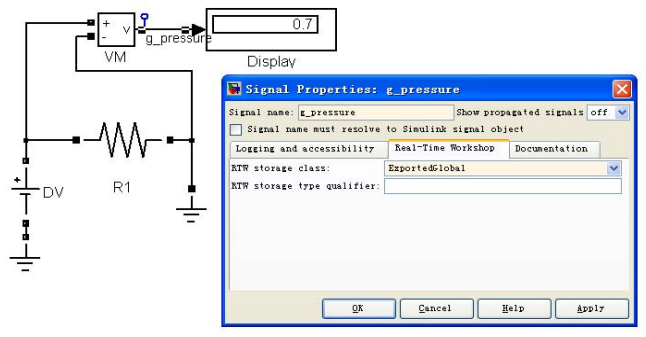

Figure 9. Signal Properties setting chart

as shown in Figure.9 the signal is set to global variables RTW automatically generated code is divided into two parts: one is the model code; the other is the interface code(runtime interface). The code was cut into many source code files, in order to manage this massive project, RTW also make an automatically generated file. Open the file with the $\mathrm{VC}$ and compile, and then referenced in this project all the source files which can be copied from the installation directory of Matlab, and together constituting the model code and the simulation model code. Model code, the three most critical function have been shown in Figure.10, the three functions constitute a simulation model program code to interact with the $\mathrm{VC}$ interface.

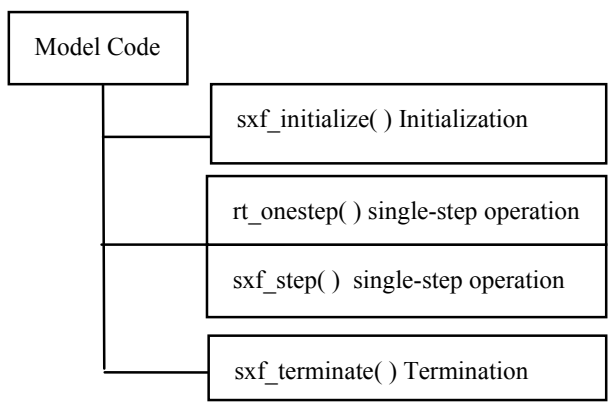

Figure 10. Fig.10 Simulate operation module interface function

\section{MODEL CODE AND THE INTEGRATION OF VC ++ PROGRAM}

Model code integration with the VC process is relatively simple, the simulation computation module source files for all involved in the project should be added to the VC program, you should do the following steps:

1) simulation module's source file of the VC program project application precompiled headers can be set up by default option, you must manually cancel the setting or compile error. Specific methods of operation are as follows: select the project in VC menu -> Select "Settings item in the dialog box. Firstly, select etr main.cpp file in the $\mathrm{C} / \mathrm{C}++$ in the Category select Precompiled Headers, as shown in Figure.11:

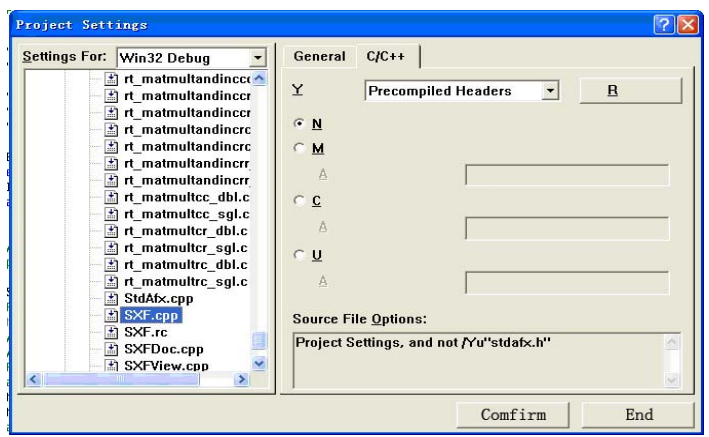

Figure 11. Project properties setting dialog box-chart

2) Predefined in the SXF.h file \# define MODEL $=* *$, $\mathrm{RT}, \mathrm{NUMST}=2, \mathrm{TID} 01 \mathrm{EQ}=1$, NCSTATES $=4, \mathrm{MT}=0$, USE RTMODEL, all of which are predefined before programming.

3) In order to call in the VC process simulation code interface functions you need to make global statements. add the following code in the application procedures in the VC class header file[4].

extern void sxf initialize (boolean T firstTime); extern void sxf_terminate (void);

extern void rt oneStep ();

The code is all the emulation functions as global functions. Semi-physical simulation software generally includes man-machine interface, data acquisition module, simulation computation module, data processing module.Flow chart of the Water Fire Fighting System's

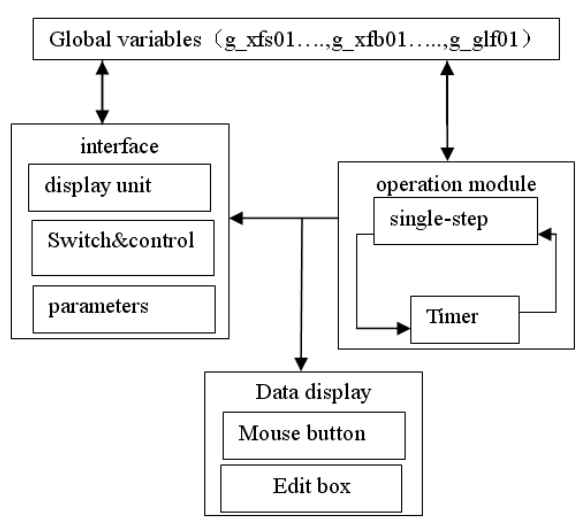

semi-

physical simulation system is shown in Figure.12.

Figure 12. Seawater firefighting system semi-physical simulation chart. 


\section{REALIZATION OF EACH MODULE}

1) Implementation of man-machine interface module. Man-machine interface module constitutes a VC program's main thread, mainly aims to complete real-time display of variables, parameters adjustment, the control parameters and procedures for operational control and other functions, to synchronize with other modules. The system variables involved in real-time include pressure of fire pumps, flux of fire pump, pressure of fire hydrant, flux of fire hydrant and status of valves and pipe status, based on the consideration of a more friendly interface, the design of fire pumps, fire hydrants, valves, message boxes, function buttons, have made full use of object-oriented encapsulation and inheritance, etc.

2) Simulation computing module. Simulation operation module of the simulation model in the RTW code based on the simulation of synchronous control and also added to meet the requirements of real-time simulation. To ensure that the entire simulation to move forward to meet the real-time requirements of the system Every $2 \mathrm{~ms}$ multimedia timer callback function to call its model code interface function rt_OneStep (model single-step operation). Real-time simulation model of computation module is to complete the calculation and also is the core of the whole system.

3) Implementation of the data processing module. Data processing module aims to complete the simulation data in real time recording and time saving. In order to meet realtime data recording, the simulation program uses a multithreaded technology. On the one hand the data suggest the mouse device with the meaning of the corresponding property, on the other through the system shows the edit box shows the attributes of each device, therefore, through the properties of the device, calculate the number of fire hydrants can work and good rates [5].

The ultimate simulation platform build through Simulink's RTW is shown in Figure.13.

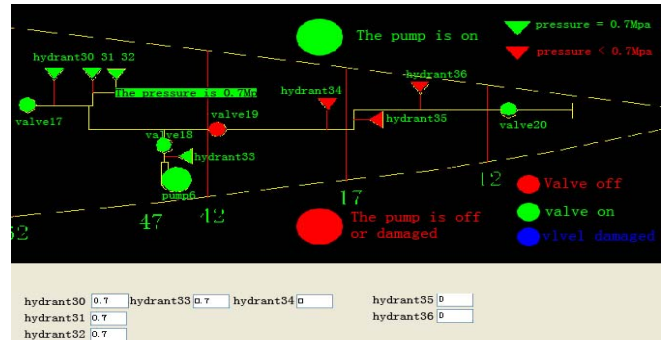

Figure 13. Seawater firefighting system simulation structural chart.

\section{SUMMARIES}

This article details with examples of scientific research which is based on Visual C + + RTW of semi-physical simulation system for rapid development method that takes full advantage of Simulink and Visual $\mathrm{C}++$. first use of Simulink visual modeling method system simulates the given water fir fighting system, and then RTW will translate Simulink simulation model into a portable embedded $\mathrm{C}++$ code, Finally, in the Visual $\mathrm{C}++$ programming environment, the simulation model can be customized into a real-time semi-physical simulation system. And also the simulation program can be easy to debug in Visual $\mathrm{C}++$ environment program.

\section{REFERENCES}

[1] ZHANG Zhao-shun; CUI Gui-xiang. Hydro-dynamics (Second Edition). Press QingHua Univ. 2002, PP: 75-77.

[2] Mathworks Company.Real-Time Windows Target User's Guide Version 2[M/CD]. U.S.PP: 213-214.

[3] Mathworks Company.Writing S-Functions Version 6 [M/CD]. U.S. PP: 32-34.

[4] David J.Kruglinski. Technology Inside Visual C++ (Fourth Edition) [M]. Press QingHua Univ. 1999, PP:156-159.

[5] Taiwan. ADVANTECH Co. Ltd. PCI-1780 User Manual [M/CD]. 2005. PP:106-107 